\title{
The Nuts and Bolts of Robotics in K-12 Classrooms: A Literature Synthesis
}

\section{Tianshi Fu}

Dr. Molly H Goldstein, University of Illinois at Urbana - Champaign

Molly H. Goldstein is an engineering design educator and researcher at University of Illinois, UrbanaChampaign. She previously worked as an environmental engineer specializing in air quality influencing her focus in engineering design with environmental concerns. Her research interests include how students approach decision making in an engineering design context. She obtained her BS in General Engineering (Systems \& Design) and MS in Systems and Entrepreneurial Engineering from the University of Illinois and $\mathrm{PhD}$ in Engineering Education from Purdue University.

\section{Prof. Holly M Golecki, University of Illinois at Urbana-Champaign}

Holly Golecki is a Teaching Assistant Professor in Bioengineering at the University of Illinois UrbanaChampaign. Prior to the move to Illinois, Holly spent 5 years as a teacher and director of robotics at a K12 private school. While there, she started a soft robotics research group for high school students. Now at the University of Illinois, she studies impacts of such pre-college engineering programs with the perspective of the classroom teacher in mind. Holly received her BS and MS in Materials Science and Engineering from Drexel University and her PhD in Engineering Sciences from Harvard University. 


\title{
Nuts and Bolts of Robotics in K-12 Classrooms: A Literature Synthesis (Work in Progress)
}

\begin{abstract}
Increasingly, over the last two decades, robotics has been used in pre-college classrooms as well as extracurricular programs as an active learning approach to engage and challenge students with STEM concepts. Recent studies have taken comprehensive approaches to understand and synthesize benefits of robotics education with respect to general effectiveness, students' learning and transfer skills, creativity and motivation, and diversity and broadening participation. Literature suggests that educational robotics is successful in enhancing effectiveness, learning, and motivation for some. However, work remains to be done in increasing the diversity of student participants and developing methods to broaden participation. This work in progress paper aims to take a step back from the established benefits in order to categorize unique ways that robotics education is presented in and out of the classroom. Results from this review provide the impetus for investigating novel approaches to incorporate robotics into the pre-engineering classroom for better impact with underrepresented groups in engineering.
\end{abstract}

Introduction

Robotics, like many STEM subjects, may appear conceptually difficult at first glance. For this reason it is thus typically taught at the post-secondary level. However, for its ability to demonstrate theoretical concepts [5], educational robotics (ER) has already been expanded into various forms serving K-12 students both in and out of the classroom. This expansion aims to diversify stagnant college STEM majors where misperceptions of difficulty has dissuaded many from participating [1]. This literature review examines how ER can (1) broadly motivate students' interests in STEM; (2) provide contextual and practical backing for otherwise abstract STEM topics; (3) facilitate learning STEM through robotics competitions; (4) help increase learning in students with disabilities; (5) boost STEM engagement by women and other underrepresented minorities and (6) incorporate the field of soft robotics to obtain additional educational benefits. The results of this literature review support a deepening awareness of the implications of robotics education at the K-12 level, reveal findings of past work and illustrate directions for future research.

Robotics to motivate STEM pathways

There stands an increasing demand for a STEM-orientated workforce. The U.S. Department of Labor [2] estimates that by 2024, there will be an increased need of 21-23\% for STEM sector jobs such as computer systems analysts and biomedical engineers. Attracting students to STEM majors and jobs, however, remains a challenge. There exists a phenomenon among high school students' who perceive that STEM subjects are difficult and therefore favor other subjects [1]. To worsen the issue, the number of students enrolled in engineering disciplines is not increasing and in some cases is decreasing [2] with especially low representation from underrepresented minorities [1] and 
female students [3]. In response to this lack of interest in STEM majors, robotics programs have been created specifically to motivate high school students toward STEM careers. The use of robotics to perform tasks is captivating [1]. Robotics competitions capture students' attention with the practicality of hands-on projects and exciting challenges while increasing students' confidence in STEM topics, such as geometry and physics [4]. This increase in comfort level with STEM topics has positively impacted these same students' perception of STEM. Leveraging this common theme of practicality, several researchers have shown that ER is an effective tool to engage K-12 students [1-4]. For example, using robotics and cooperative learning, a group of college-aged students served as instructors to middle school students in a robotics program. Mentors introduced students to robotics concepts along with key STEM elements of critical thinking and self-efficacy. This resulted in an increase not only in STEM interests, but other problem solving skills from participants of both genders [1].

The increased interest in STEM is echoed by students in research by Leonard et al [2]. In this work, a group of 28 students participated in a two-week summer camp on robotics and game design. All students, including female and under-represented minority groups exhibited a high level of engagement with high pre- and post-activity STEM attitude scores. In work by Barco et al [3], facilitators focused specifically on improving female participation through teaching robotics with social applications, to better align with female students agency beliefs. The experiment was conducted with participants consisting of all female high school students. Results showed that female students have a significantly higher preference for social robotics activities. Example activities included robots built to care for the elderly or children with disabilities rather than traditional task orientated robot applications. This work indicates that the benefits for STEM encouragement is not generated by robotics alone. Instead, it is specific applications of robotics that can be used to attract underrepresented minorities. The work summarized here illustrates that intentional curricular design for ER programs may be an effective tool to increase student diversity in the STEM fields.

Robotics as a vehicle to strengthen STEM concepts

Previous ER implementations and research suggests the practicality of using a robotics curriculum in order to promote meaningful learning of STEM concepts [5]. These ER implementations have transformed traditional STEM education processes by teaching theoretical concepts through concrete robotics experiences. While some STEM subjects have laboratory, activities integrated into the course, many, such as mathematics, traditionally do not. Consequently, it is often difficult for students to grasp traditional, abstract mathematics concepts without tangible examples. Research has suggested that technology may be a powerful tool for learning and developing math transfer skills [10]. Many current ER studies focus on enhancing transfer skills through the demonstration of connections between abstract concepts and tangible examples in robotics [8]. Learning transfer occurs when students apply conceptual knowledge in a new context. For instance, in one study, students developed a deeper understanding of ratios through building gears and pulleys systems commonly used in robots [5]. In another example, Brockington et al. [7] used a robotic vehicle to help students understand concepts of breaking distance and friction by experimenting with different types of wheels. Similarly, Cuperman and 
Verner [6] found that students were able to develop and transfer bio-mechanical skills by matching robotic components to similar biological assemblies as many robots are mechanically analogous to living organisms. Furthermore, mathematical concepts of proportions and ratios were successfully introduced to a group of students as young as fifth grade [8]. In this study, while both control (traditional textbook lessons) and experimental groups performed similarly on numerical computation problems, the experimental group exhibited far superior learning transfer skills when tested on mathematical word problems [8]. The experimental group performed better when they needed to extract mathematical meaning from sentences [8]. This work suggests that ER may be an effective tool to break down the barriers in classical STEM courses currently keeping some students from majoring in these subjects.

In addition to math and science subjects, ER can provide an effective way for students to learn computer science, as robotics require programs to operate. One robotics implementation involved elementary students working with a visual programming language, writing and running programs in order to control the robot's motion [9]. This immediate feedback from code to robot motion captured students' attention and allowed for otherwise complex ideas to be understood and used by young students [9]. Studies included in this section demonstrate the capability of ER in STEM classrooms, suggesting the inclusion of robotics in K12 curricula can be beneficial for students and their comprehension of conceptual knowledge. Despite overwhelming reports of positive impact brought on by adding robotics to STEM subjects, instructors still treat robotics as an add-on to the course instead of building the course from ground up with robotics in mind. Robotics as a permanent fixture in classroom, may increase STEM comprehension and engagement. In addition to the traditional classroom setting, a more common method of using robotics as the foundation of learning STEM knowledge is in the competitive setting which has become popular among students and enthusiasts.

Robotics competition

One of the most common robotics activities in a K-12 context is competition. FIRST LEGO League (FLL) attracted more than 100,000 participants just two years after its inception and has quickly expanded to over 70 countries worldwide [12]. This rapid growth is partly due to the increasing demand for a STEM educated workforce. Robotics competitions are believed to foster interest in STEM which can lead students to pursue related professions [15]. This competitive setting fosters a rigorous environment for students to learn various STEM concepts through designing and building robots [11] as well as an opportunity to practice problem-solving and decision-making skills [12]. There are two major groups of robotics competitions: FLL and VEX Robotics Competition. In both programs, students work cooperatively with each other to build robots to complete goal-oriented tasks. An exploration of middle school students who participated FLL for two consecutive years showed that students gained insight into the iterative engineering process of design, construction and experimentation where some even actively learned new concepts to resolve issues through physical prototyping [13]. These results are backed by similar research on FLL participants in which both students, assisting teachers, and mentors reported gains in STEM competence and social skills from working team environments [12]. Other research focusing specifically on the impact of competing in FLL and mathematics produced some contrasting results. Teams that used math extensively in their robot designs had a polarizing performance in the competition with scores at both ends of the spectrum [11]. This 
suggests that while the competitive environment can be a catalyst for learning, use of fundamentals does not always translate to design successes. Despite lack of success in FLL competition, math transfer tests showed improvements as well as increases in mathematics interest from students [11]. Research on VEX companions yielded similar results [14, 15]. In the context of European educational systems, ER and VEX robotics competition serve as good supplements for STEM learning activities [14]. However, investigation into VEX robotics competitions revealed a significant gender disparity in its participants [15]. While both male and female students have equal STEM capabilities, Sullivan and Umaschi Bers [15] suggest that the competitive environment is an opportunity to boost female students' confidence and technical expertise to further their development in STEM. In contrast, other researchers suggest that the effects of a competitive setting are not entirely beneficial [15]. Fear of failure and competition anxiety may negatively impact students' motivation to explore further STEM knowledge [15]. The VEX platform recently form the Girl Powered Initiative to aid in specifically empowering female participants through scholarships, workshops, and dedicated programming. Long-term outcomes from this program are an area for future research. Despite some possible drawbacks, STEM learning successes cited here show that robotics competitions can provide an environment for students to engage and learn outside of the classroom.

Using robotics for assisting students with disabilities

This review has discussed robotics in K-12 classrooms as well as robot competitions. In addition, research into how ER can aid in learning specifically for students with developmental and learning disabilities has provided promising results. The interactive mediums of robotics assist in overcoming challenges and may promote further engagement with STEM [16]. As robotics kits become more accessible, they have been employed in the diagnosis and treatment of autism spectrum disorder (ASD) [17]. Acknowledging the potential of robotics to engage students with disabilities, research by Knight et al, [16] studied the impacts of a summer robotics camp for students with ASD and social anxiety. By making robotics a shared interest, participants reported a significant reduction in anxiety. Students with ASD who previously struggled with social cues, demonstrated understanding the nuanced purpose behind gestures in social interactions through programming specific behaviors for a robot [17]. The positive impact of robotics is also reported by another study of a student with ASD coupled with a severe behavior disorder [16]. After interacting with a robotics-based intervention, the participant showed great interest in the subject and exhibited more positive behaviors in the experimental period [16]. ER may play a role in engaging students with disabilities to further allow for academic developments through robotics concepts and activities.

Application of ER in a program for students with Down syndrome also yielded positive results [18]. As seen previously, the use of robotics was able to capture the attention of these students and enabled them to learn in a cooperative environment. Moreover, assessments suggested that students with Down syndrome exhibited improvements in cognitive functions such as visuospatial working memory and mental planning after completing a robotics challenge [18]. While recent work indicates that robotics is a viable tool to assist special needs students in overcoming their leaning and developmental challenges, it is still at its infancy. Current research has an extremely limited sample size and study period. Furthermore, there also exists a fair amount of heterogeneity in the studies, where participants have different age, cognitive profiles and other 
co-occurring disorders [18]. The potential impact of ER for students with developmental and learning disabilities, warrants further study and funding.

STEM engagement of women and other underrepresented groups

Despite having vast potential in education, the main beneficiaries of ER are male and predominantly white. Many forms of ER examined in previous sections appear to be less effective in engaging women and other underrepresented groups. For example, over $70 \%$ of participants in robotics competitions such as FLL are male [30]. Effectiveness of STEM engagement is further diminished by the division of work in the competition groups. One study highlighted that male students usually take part in the design and technical side whereas female students tend to fulfill more social roles such as marketing, fundraising, communications and community service [30]. However, this issue is less pronounced in small groups where technical roles are more available to women [33]. In general, girls appear to lose interest in STEM much faster than boys even with interventions as they progress through high school $[23,26]$. The situation is similar for other underrepresented groups in STEM. Only 11\% of all college freshmen students are enrolled in engineering and about $10 \%$ of those are Hispanic students [28, 30]. Because choice of major in college has a significant impact on their career selections, this has resulted in lack of diversity in the STEM workforce [24]. A diverse STEM workforce is shown to promote creativity and increase profits [25]. To challenge the racial and gender imbalance in STEM, human and social-centered ER curriculums have been developed. This type of ER aims to align its theme to female and underrepresented minorities' interests.

\section{Soft robotics}

One instance of human and social centered ER is soft robotics. According to Jackson et al., unlike traditional robotics which focuses on mechanical and electrical engineering, soft robotics draws more from disciplines such as biomedical, agricultural and materials engineering [30]. These human-centered and societal relevant majors have much higher female enrollment than those associated with traditional robotics [26]. Moreover, a study has revealed that young girls are significantly more interested in robotics when there are identifiable connections to human benefits [31]. Consequently, building robots from soft materials with broad human centered applications, may shine a different light on robotics and attracts more female participation.

Use of soft, deformable materials to construct robots has become more popular in the ER context due to several advantages over its traditional rigid counterpart. These robots are constructed from elastomers or textiles and use cable-based or pneumatic actuation methods. ER for K12 students usually does not involve complex topics such as control theories or sensing strategies. As a result, it can be difficult for students to control robots without such knowledge [19]. Furthermore, common robotics kits such as FLL and VEX can have a high barrier of entry due to price. In contrast, simple soft robots such as pneumatic powered silicone manipulators can be inexpensive to build with little or no requirement for computer programming knowledge [20]. In this case motion can be accomplished with mechanical programming [19]. One example of mechanical programming is to orient fiber reinforcements in a specific direction to control the motion of a composite soft actuator. Soft robotics competitions were conducted yearly from 2015-2018 by the Soft Robotics Toolkit [32]. The stated goal was to address problems identified 
by participants with soft systems in order to expand soft robotics applications [21]. Group of students were tasked to build robots using low modulus materials [19]. To accomplish this goal, students leveraged the soft body morphology of materials used. Post competition surveys showed that students enjoyed the experience while gaining robotics and mechanics knowledge [19]. In an entry into the Soft Robotics Toolkit competition, Sardesai et al employed gelatinbased materials to construct edible and biodegradable pneumatic manipulators [22]. The edible actuator manages to entice interest of students and the biodegradable functionality extends its application into the medical field [22]. Compared to rigid robots, use of soft robotics in education is still at its infancy however, with its accessibility and unique material properties, soft robotics have a promising future in educational robotics.

Future work

Robotics attracts students in the classroom environment and promotes learning of traditionally abstract concepts to attract broad confidence and participation. Robotics also serves as a driving force for STEM education outside of the classroom where competitions encourage learning of technical knowledge as well as working as a team. Potential curriculum development around applications of robots outside traditional task oriented devices, may promote interest from a more diverse group of students. Furthermore, ER shows potential in assisting students with learning and developmental disabilities. As a tool for education, there is an abundance of literature that points toward the possible benefits robotics can provide and newer research that shows robotics may be attractive to females and underrepresented minorities given new applications and materials. Soft robotics shows promise in providing a more accessible entry for K12 classrooms. Future work will investigate the use of robotics in the classroom to understand opportunities for broad participation in K12 implementations.

\section{References}

[1] P. Mosley, G. Ardito and L. Scollins, "Robotic Cooperative Learning Promotes Student STEM Interest", American Journal of Engineering Education, vol. 7, no. 2, pp. 117-128, 2016.

[2] J. Leonard, A. Buss, A. Unertl and M. Mitchell, "USING ROBOTICS AND GAME DESIGN TO PROMOTE PATHWAYS TO STEM", in Annual Meeting of the North American Chapter of the International Group for the Psychology of Mathematics Education, Tucson, AZ, 2016, pp. 1487-1494.

[3] A. Barco, R. Walsh, A. Block, K. Loveys, A. McDaid and E. Broadbent, "Teaching Social Robotics to Motivate Women into Engineering and Robotics Careers", in $201914^{\text {th }}$

[4] ACM/IEEE International Conference on Human-Robot Interaction (HRI), Daegu, Korea (South), 2019, pp. 518-519.

[4] B. Brand, M. Collver and M. Kasarda, "Motivating Students With Robotics", The Science Teacher, vol. 75, no. 4, pp. 44-49, 2008. 
[5] E. M. Silk and C. D. Schunn, "Using robotics to teach mathematics: Analysis of a curriculum designed and implemented", in American Society for Engineering Education, Pittsburgh, PA, USA, 2008.

[6] D. Cuperman and I. M. Verner, "Fostering Analogical Reasoning Through Creating Robotic Models of Biological Systems", Journal of Science Education and Technology, vol. 28, no. 2, pp. 90-103, 2019.

[7] G. Brockington, M. Schivani, C. Barscevicius, T. Raquel and M. Pietrocola, "Using robotics in kinematics classes: exploring braking and stopping distances", Physics Education, vol. 53, no. 2, 2018. Available: 10.1088/1361-6552/aaa09e.

[8] A. Ortiz, "Examining Students' Proportional Reasoning Strategy Levels as Evidence of the Impact of an Integrated LEGO Robotics and Mathematics Learning Experience", Journal of Technology Education, vol. 26, no. 2, pp. 46-73, 2015.

[9] M. Friebroon Yesharim and M. Ben-Ari, "Teaching Computer Science Concepts Through Robotics to Elementary School Children", International Journal of Computer Science Education in Schools, vol. 2, no. 3, 2018. Available: 10.21585/ijcses.v2i3.30.

[10] S. Papert, Mindstorms: children, computers, and powerful ideas. Brighton: Basic Books, Inc., 1980.

[11] E. M Silk, R. Higashi and C. D Schunn, "Resources for Robot Competition Success: Assessing Math Use in Grade-School-Level Engineering Design", in American Society for Engineering Education, Vancouver, BC, Canada, 2011.

[12] N. Arís and L. Orcos, "Educational Robotics in the Stage of Secondary Education: Empirical Study on Motivation and STEM Skills", Education Sciences, vol. 9, no. 2, p. 73, 2019. Available: $10.3390 /$ educsci9020073.

[13] F. KALOTI-HALLAK, M. ARMONI and M. BEN-ARI, "The Effect of Robotics Activities on Learning the Engineering Design Process", Informatics in Education, vol. 18, no. 1, pp. 105129, 2019. Available: 10.15388/infedu.2019.05.

[14] I. Alvarez Caro, "VEX Robotics: STEM Program and Robotics Competition Expansion into Europe", in Eurobot Conference 2011, Prague, Czech Republic, 2011, pp. 10-16.

[15] A. Sullivan and M. Umaschi Bers, "VEX Robotics Competitions: Gender Differences in Student Attitudes and Experiences", Journal of Information Technology Education: Research, vol. 18, pp. 097-112, 2019. Available: 10.28945/4193. 
[16] V. Knight, J. Wright and A. DeFreese, "Teaching Robotics Coding to a Student with ASD and Severe Problem Behavior", Journal of Autism and Developmental Disorders, vol. 49, no. 6, pp. 2632-2636, 2019. Available: 10.1007/s10803-019-03888-3.

[17] J. Kaboski et al., "Brief Report: A Pilot Summer Robotics Camp to Reduce Social Anxiety and Improve Social/Vocational Skills in Adolescents with ASD", Journal of Autism and Developmental Disorders, vol. 45, no. 12, pp. 3862-3869, 2014. Available: 10.1007/s10803014-2153-3.

[18] S. Bargagna et al., "Educational Robotics in Down Syndrome: A Feasibility Study", Technology, Knowledge and Learning, vol. 24, no. 2, pp. 315-323, 2018. Available: 10.1007/s10758-018-9366-z.

[19] X. Yu, S. Nurzaman, U. Culha and F. Iida, "Soft Robotics Education", Soft Robotics, vol. 1, no. 3, pp. 202-212, 2014. Available: 10.1089/soro.2014.0009.

[20] B. Finio, R. Shepherd and H. Lipson, "Air-powered soft robots for K-12 classrooms", in 2013 IEEE Integrated STEM Education Conference (ISEC), Princeton, NJ, USA, 2013.

[21] D. P. Holland, G. J. Bennett, G. M. Whitesides, R. J. Wood and C. J. Walsh, "The 2015 Soft Robotics Competition", IEEE Robotics \& Automation Magazine, vol. 23, no. 3, pp. 25-27, 2016.

[22] A. Sardesai et al., "Design and Characterization of Edible Soft Robotic Candy Actuators", MRS Advances, vol. 3, no. 50, pp. 3003-3009, 2018. Available: 10.1557/adv.2018.557.

[23] A. Gomoll, C. Hmelo-Silver, S. Šabanović and M. Francisco, "Dragons, Ladybugs, and Softballs: Girls' STEM Engagement with Human-Centered Robotics", Journal of Science Education and Technology, vol. 25, no. 6, pp. 899-914, 2016. Available: 10.1007/s10956-0169647-z.

[24] A. Sullivan and M. Bers, "Investigating the use of robotics to increase girls' interest in engineering during early elementary school", International Journal of Technology and Design Education, vol. 29, no. 5, pp. 1033-1051, 2018. Available: 10.1007/s10798-018-9483-y.

[25] A. Sullivan and M. Bers, "The Impact of Teacher Gender on Girls' Performance on Programming Tasks in Early Elementary School", Journal of Information Technology Education: Innovations in Practice, vol. 17, pp. 153-162, 2018. Available: 10.28945/4082. 
[26] A. Jackson, N. Mentzer and R. Kramer-Bottiglio, "Pilot analysis of the impacts of soft robotics design on high-school student engineering perceptions", International Journal of Technology and Design Education, vol. 29, no. 5, pp. 1083-1104, 2018. Available: 10.1007/s10798-018-9478-8.

[27] J. Fong and H. Cheng, "C-STEM Girls Computing and Robotics Leadership Camp", in ASEE Annual Conference \& Exposition, Indianapolis, IN, 2014.

[28] T. Yuen, L. Ek and A. Scheutze, "Increasing participation from underrepresented minorities in STEM through robotics clubs", in IEEE International Conference on Teaching, Assessment and Learning for Engineering (TALE), Bali Dynasty Resort, Kuta, Indonesia, 2013.

[29] M. R. Anderson-Rowland, A. A. Rodriguez and A. E. Grierson, "Getting the attention of underrepresented minority community college students to consider engineering as a career," 2015 IEEE Frontiers in Education Conference (FIE), El Paso, TX, 2015, pp. 1-8. doi: 10.1109/FIE.2015.7344328

[30] A. Jackson, J. Zhang, R. Kramer and N. Mentzer, "Design-based Research and Soft Robotics to Broaden the STEM Pipeline (Work in Progress)", in 2017 ASEE Annual Conference \& Exposition, Columbus, Ohio, 2017.

[31] B. S. Terry, B. N. Briggs and S. Rivale, "Work in progress: Gender impacts of relevant robotics curricula on high school students' engineering attitudes and interest," 2011 Frontiers in Education Conference (FIE), Rapid City, SD, 2011, pp. T4H-1-T4H-3.

doi: 10.1109/FIE.2011.6143090

[32] "Soft Robotics Toolkit", Softroboticstoolkit.com, 2020. [Online]. Available: https://softroboticstoolkit.com/.

[33] Center for Youth and Communities. "Cross-program evaluation of the first tech challenge and the first robotics competition," Heller School for Social Policy and Management, Brandeis University, Waltham, MA, 2011. 